\title{
Inertial mass from Unruh temperatures
}

\author{
J. Giné ${ }^{\dagger}$ and M.E. McCulloch ${ }^{\ddagger}$ \\ † Departament de Matemàtica, Universitat de Lleida, \\ Catalonia, Spain. \\ $\ddagger$ SMSE, Plymouth University - Plymouth, PL4 8AA, UK, EU \\ E-mails: gine@matematica.udl.cat and mike.mcculloch@plymouth.ac.uk
}

\begin{abstract}
It has been proposed that inertia can be explained as follows: when objects accelerate in one direction a Rindler horizon forms in the other direction, suppressing Unruh radiation on that side, and producing a net Unruh radiation pressure that always opposes the acceleration, just like inertia. So far this model has predicted masses over twice those expected. In this paper an error in this model is corrected so that its prediction improves to within $29 \%$ of the expected Planck mass. It is also shown that inertial mass may be understood qualitatively by applying Carnot's principle and entropy to Unruh temperatures, so that the work needed for inertia comes from the difference in the Unruh temperatures seen by the accelerated object and the cosmos. This implies that highly-accelerated systems may emit heat in a new way.
\end{abstract}

Keywords: Cosmology; Unruh radiation; Hubble-scale Casimir effect; inertial mass.

PACS 98.80.-k - Cosmology

$P A C S$ 04.62.+v - Quantum fields in curved spacetime

PACS 06.30.Dr - Mass and density 


\section{Introduction}

Hawking (1975) showed that black holes can emit thermal radiation. This effect is known as Hawking radiation and happens because the event horizon of a black hole can separate paired virtual particles. A similar effect happens for an accelerated particle. On the side that the particle is accelerating away from, a dynamical (Rindler) event horizon appears, see Rindler (2001) which produces radiation, the Unruh (1976) radiation, which is similar to Hawking radiation. Therefore an accelerated particle perceives a warm background whereas a non-accelerated particle will see a cold background with no radiation.

In McCulloch (2013) a new model for inertia was suggested: for an accelerated particle the Unruh radiation becomes non-uniform because the Rindler event horizon reduces the energy density in the direction opposite to the acceleration vector (due to a Rindler-scale Casimir effect). Therefore there is an imbalance in the momentum transferred by the Unruh radiation and this produces a force which is always opposed to the acceleration, just like inertia. McCulloch (2007) also proposed a modification of this basic inertial mass due to the distant Hubble horizon, which is called Modified inertia due to a Hubble-scale Casimir effect (MiHsC), also called Quantized Inertia. $\mathrm{MiHsC}$ predicts that only Unruh waves that fit exactly into twice the Hubble diameter are allowed, so that an increasingly greater proportion of the Unruh waves are disallowed as accelerations decrease and these waves get longer, leading to a new gradual loss of inertia as acceleration reduces. In MiHsC the standard inertial mass $\left(m_{i}\right)$ becomes modified to $m_{I}$ as

$$
m_{I}=m_{i}\left(1-\frac{\beta \pi^{2} c^{2}}{|a| \Theta}\right) \sim m_{i}\left(1-\frac{2 c^{2}}{|a| \Theta}\right),
$$

where $a$ is the total acceleration of the test particle with respect to other masses, $\beta$ appears in Wien's constant and has the value $\beta=0.2$, and $\Theta$ is the Hubble diameter $\Theta=2 c / H_{0}=2 R_{U}$. This model has been used to explain the galaxy rotation problem (ad hoc arrangements of dark matter can also explain this) and to explain the observed cosmic acceleration and other astronomical anomalies, see McCulloch (2007, 2010, 2012). MiHsC does violate the equivalence principle in that $m_{i} \neq m_{g}$. However, this violation could not have been seen in experimental tests. Torsion balance tests of the equivalence principle are far more accurate forms of Galileo's experiment, in 
which he is supposed to have dropped two balls of different mass off a tower and measured their drop time to be equal. In torsion balance tests the two balls are located at both ends of a cross bar, suspended from its mid-point by a wire, and they gravitate or fall horizontally towards distant masses, for example the Sun. If there is no twist in the wire, the equivalence principle is not taken to be violated. However, the extra acceleration predicted by $\mathrm{MiHs}$ is independent of the mass, so it predicts an extra acceleration which is equal for both balls, resulting in no twist in the wire and no apparent experimental violation of equivalence.

In this note we correct a mistake in the derivation in McCulloch (2013) where the mechanism for inertia was given, and so produce a more successful result. It is also shown that inertia can be qualitatively understood by applying Carnot's principle and entropy to Unruh temperatures, and that this suggests a possible thermal laboratory test.

\section{McCulloch's model for inertia}

When an object accelerates, for example to the right, a dynamical Rindler horizon forms to its left (see the left hand black curve in Figure 1) and the object sees Unruh radiation all around it (Unruh, 1976). The new suggestion by McCulloch (2013) is that the Rindler horizon reduces the Unruh radiation on, in this case, the left hand side because only Unruh wavelengths that fit into the distance between the object and the horizon are allowed. This can be thought of, and has the same form, as a Rindler-scale Casimir effect. In contrast, the radiation on the right hand side is only slightly reduced by a Hubble-scale Casimir effect due to the far-off cosmic horizon. This produces an imbalance in the radiation pressure on the object, and a net force that always opposes acceleration, like inertia.

However, the previous derivation included a factor of two error and so overpredicted the inertial mass. The derivation is corrected here. A single particle (the black circle in Figure 1) accelerates to the right and sees a radiation pressure incident on its surface of

$$
F=\frac{u A}{3}
$$

where $\mathrm{u}$ is the radiation energy density, $\mathrm{A}$ is the surface area intercepting this radiation. Now we derive the net difference between the force from the 
left and the right, so we consider an arbitrary line through the particle at an angle $\theta$ (the long-dashed line in Figure 1) and calculate the net force on the particle along this line from both directions and take its component along the $\mathrm{x}$ axis

$$
d F_{x}=\frac{u_{\text {left }} A \cos \theta}{3}-\frac{u_{\text {right }} A \cos \theta}{3}
$$

Now a Rindler horizon forms to the left at a distance $c^{2} / a$ from the particle, where a is its acceleration and the cosmic horizon is a radial distance $\Theta / 2$ away. In McCulloch (2013), as in McCulloch (2007, 2012), the cosmic horizon was assumed to have a diameter of $2(\Theta / 2)=\Theta$ so the radiation coming from the direction of acceleration (right) was subject to a Hubblescale Casimir effect so that its radiation energy density $(u)$ was modified as $u_{\text {right }}=u(1-\lambda /(4 \Theta))$ where $\lambda$ is the peak wavelength of the Unruh spectrum. Now, to be consistent, the radiation coming from the opposite direction must be subject to a Rindler-scale Casimir effect with the event horizon having now a diameter of $2 c^{2} /(a \cos \theta)$ (where $a \cos \theta$ is the component of the acceleration in the direction $\theta)$ so that we now have $u_{\text {left }}=u\left(1-\frac{\lambda}{4\left(2 c^{2} /(a \cos \theta)\right)}\right)$.

Recomputing the calculations then we find that the net force along the line in the $\mathrm{x}$ direction is

$$
d F_{x}=\frac{u \lambda A \cos \theta}{3}\left(\frac{1}{4 \Theta}-\frac{a \cos \theta}{8 c^{2}}\right) .
$$

We now integrate the contributions from all angles. Integrating from $\theta=0$ to $\theta=\pi / 2$ then double this to get the result for the $\mathrm{x}, \mathrm{y}$ plane and then we integrate along the azimutal angle $\phi$ from 0 to $\pi$ to calculate the total force:

$$
F=2 \times \frac{u \lambda A}{3} \int_{0}^{\pi} \int_{0}^{\pi / 2}\left(\frac{\cos \theta}{4 \Theta}-\frac{a \cos ^{2} \theta}{8 c^{2}}\right) d \theta d \phi
$$

This becomes

$$
F=\pi \frac{2 u \lambda A}{3}\left(\frac{1}{4 \Theta}-\frac{\pi a}{32 c^{2}}\right) .
$$

Now we can neglect the first term, which is tiny if we assume typical accelerations (a) found on Earth and we have

$$
F=-\frac{\pi^{2} u \lambda A a}{48 c^{2}} .
$$


Since $u=E / V=h c / \lambda V$, where $V$ is volume, then

$$
F=-\frac{\pi^{2} h A a}{48 c V}
$$

Assuming for simplicity that the particle is a cube, the ratio $A / V=x^{2} / x^{3}=$ $1 / x$ and considering its side is the Planck distance $l_{p}=1.616 \times 10^{-35} \mathrm{~m}$ as $x$, then equation (8) becomes

$$
F=-\frac{\pi^{2} h a}{48 c l_{p}},
$$

and the inertial mass is $m_{i} \sim \pi^{2} h /\left(48 \mathrm{cl}_{p}\right) \sim 2.799 \times 10^{-8} \mathrm{~kg}$ which is $29 \%$ greater than the Planck mass $m_{p}=2.176 \times 10^{-8} \mathrm{~m}$ in closer agreement than in McCulloch (2013) where the result was 2.53 times the Planck mass (Note that another error in the previous paper is that the Unruh radation is said to be 'anisotropic', whereas in fact the author meant 'isotropic').

\section{An qualitative thermal model}

It is possible to think about $\mathrm{MiHsC}$ in a thermodynamical way rather than thinking in terms of waves and how they fit into horizons or the Hubble scale. For example, Eq. (1) can also be written using a ratio of accelerations:

$$
m_{I}=m_{i}\left(1-\frac{a_{H}}{|a|}\right)
$$

where $a_{H}$ is the minimum acceleration predicted by MiHsC (McCulloch, 2007, 2010) which is the background cosmic acceleration, and - a - is still the local acceleration relative to nearby masses. Since the Unruh temperature is proportional to the acceleration, this can be written, as shown by Giné (2012), also as

$$
m_{I}=m_{i}\left(1-\frac{T_{H}}{T_{L}}\right)
$$

where $T_{H}$ is the Unruh temperature of the background due to the minimum acceleration predicted by MiHsC in McCulloch (2010) and $T_{L}$ is the Unruh temperature seen by the accelerated object in question.

Here we will consider Eq. 11 further. Imagine a hot reservoir at temperature $T_{1}$ and a cold reservoir at temperature $T_{2}$. According to Carnot's Principle 
work can only be done when there is a difference in temperature, so that heat can flow from the hot to the cold. Clausius and Kelvin showed that the maximum work that can be extracted from such a setup is given by

$$
W=Q_{1}\left(1-\frac{T_{2}}{T_{1}}\right)
$$

where $W$ is the work done, and $Q_{1}$ is the heat flux from the hot object. The point is that Eq. 12 looks very much like Eq. 11 and this implies that the energy required to produce inertial mass could also be thought of as the work done by the leakage of Unruh heat from the accelerated reference frame to the background cosmic reference frame. Using $E=m c^{2}$ we can write both $W$ and $Q_{1}$ as masses

$$
m_{W}=m_{1}\left(1-\frac{T_{2}}{T_{1}}\right)
$$

This equation means that if the acceleration of a mass reduces towards the cosmic acceleration, about $6.7 \times 10^{-10} \mathrm{~m} / \mathrm{s}^{2}$, then its Unruh temperature $T_{L}$ reduces towards the Unruh temperature of the cosmos $T_{H}$. As this point no work can be extracted from the difference in temperatures and so inertial mass becomes zero, as is the case for $\mathrm{MiHsC}$ as already published. In the original model, this occurs geometrically as the Unruh waves do not fit into the Hubble scale, cannot 'in principle' be observed and, following Mach's principle they dissapear. In this thermal formulation it occurs because no work can be extracted from the zero, or very small, temperature difference. This keeps the perceived Unruh temperature of any body $T_{L}$ above the cosmic temperature.

If the acceleration reduces below the minimum acceleration allowed by $\mathrm{MiHsC}$, the Unruh temperature seen by the object falls below the background cosmic temperature, the large scale average Unruh temperature, and so heat energy can flow into the Unruh temperature of the object to this can be converted to energy to accelerate it again.

It is clear that the background temperature required here is not the environmental temperature, since Unruh temperatures, even for faster terrestrial accelerations, are only of the order $10^{-20} \mathrm{~K}$ and the environmental background is always hotter than this, so there could be no heat loss from the accelerated object and therefore no inertia. The temperature of the background must be the Unruh temperature of the Hubble boundary, due to the cosmic acceleration. 


\section{Discussion}

To discuss this in a more realistic context: if we push a ball in deep space, it accelerates, so its Unruh temperature increases above the Unruh temperature of the cosmos. The accelerated ball's Unruh temperature is not immediately visible to onlookers, it is only detected by the ball, so let us call this kind of heat: Uheat, after Unruh. It is proposed here that this Uheat is available for work. It can be moved from the Uhot ball to the Ucolder surroundings. This process is possible because it increases entropy since afterwards the Utemperature is more uniform in the cosmos. Also, this heat exchange allows work to be extracted from the system and it is proposed here that this produces the force that you feel pushing back at you as you push the ball. It should be said that this thought experiment assumes that specific heat is positive. This is usually the case, but may not be for the strong gravity of a black hole for example (Myung, 2003). Here we have to assume that we are not in a strong gravitational field.

To see why this should push the ball backwards against your acceleration of it, entropy must be considered. If the force pushed equally all around or forward then it would increase the difference between the Utemperature of the ball and its surroundings, decreasing the entropy. Only, if the force pushes back against the imposed acceleration to reduce the acceleration you have applied will it decrease the Utemperature difference, and increase the entropy. In this qualitative way the second law of thermodynamics provides an explanation for inertia and also its direction.

A test for this thermal approach to $\mathrm{MiHsC}$ would be challenging since the Utemperature of most terrestrial systems is tiny. For an acceleration of $9.8 \mathrm{~m} / \mathrm{s}^{2}$ for example the Utemperature is $T=\hbar a / 2 \pi c k=4 \times 10^{-20} \mathrm{~K}$.

However, one could look for anomalous non-frictional heating or radiation observed close to highly accelerated objects, possibly in water which would absorb the heat and retain it. One clear example is Beversluis et al. (2013) who hugely accelerated electrons around gold nanotips and detected the emis-

sion of anomalous infrared radiation. Smolyaninov (2008) proposed that this radiation was Unruh radiation. 


\section{Conclusion}

An earlier paper showed that inertial mass can be derived by assuming that inertia is caused by the formation of a Rindler horizon behind an object as it accelerates, which suppresses Unruh waves on that side of the object and pushes it back again the acceleration. In this paper the derivation of this model is corrected.

It is also suggested that inertia can be understood as being an attempt by an accelerated system to equalise Unruh temperatures between the background cosmic value and that due to its own accelerations. In this way inertia may also be understood using entropy, but only qualitatively so far. A test is suggested that looks for anomalous heat leaking out of highly-accelerated systems.

\section{Acknowledgements}

The first author is partially supported by a MINECO/ FEDER grant number MTM2014-53703-P and an AGAUR (Generalitat de Catalunya) grant number 2014SGR 1204.

\section{References}

Beverluis, M.R., A. Bouhelier, L. Novotny.: Continuum generation from single gold nanostructures through near-field mediated intraband transitions, Physical Review B, 68, 115433 (2003).

Giné, J.: The holographic scenario, the modified inertia and the dynamics of the universe, Modern Physics Letters A, 27, no. 34, 1250208 (2012).

Hawking S.W.: Particle creation by black holes, Commun. Math. Phys. 43, 199-220. Erratum-ibid 46 (1976) 206-206 (1975).

McCulloch, M.E.: Modelling the Pioneer anomaly as modified inertia, Mon. Not. Roy. Astron. Soc. 376, 338-342 (2007).

McCulloch, M.E.: Minimum accelerations from quantised inertia, Europhys. Lett., 90, no. 2, 29001 (2010).

McCulloch, M.E.: Testing quantised inertia on galactic scales, Astrophysics and Space Science, 342, no. 2, 575-578 (2012). 
McCulloch, M.E.: Inertia from an asymmetric Casimir effect, Europhys. Lett., 101, no. 3, 59001 (2013).

Myung, Y.S., 2003. Logarithmic corrections to the Bekenstein-Hawking entropy for five-dimensional black holes and de Sitter spaces. Phys. Lett. B., 574, 3-4, 289-295.

Rindler, W.: Relativity, Special, General and Cosmological, Oxford University Press (2001).

Smolyaninov, I.I.: Photoluminescence from a gold nanotip in an accelerated reference frame (2013) arxiv: cond-mat/0510743.

Unruh, W.G.: Notes on black hole evaporation, Phys. Rev. D 14, 870-892 (1976).

\section{Figures}

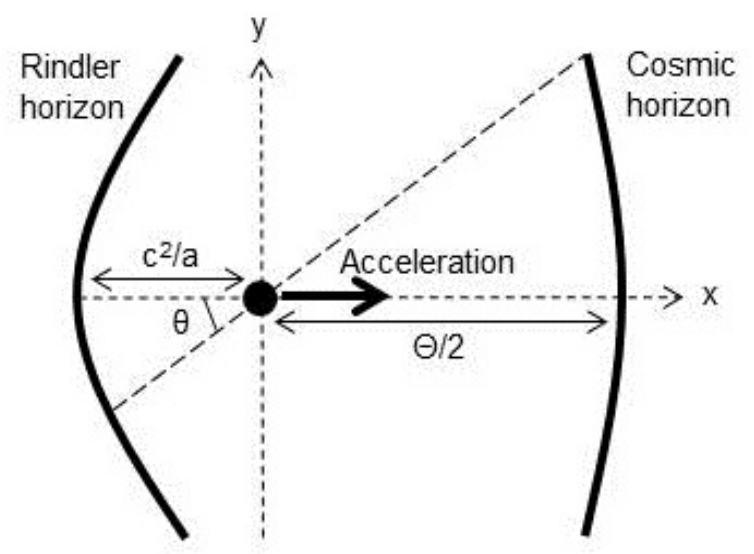

Figure 1. Schematic showing a particle (black circle) with acceleration 'a' to the right, which sees a Rindler horizon a distance $c^{2} / a$ to its left (the left hand black curve) and the cosmic horizon a far greater distance $\Theta / 2$ to its right (the right hand black curve). This produces an asymmetry in the Unruh radiation pressure that pushes the particle to the left against its acceleration: a model for inertia. The $\theta$ shows an arbitrary angle of integration in the $\mathrm{x}$, y plane. 